\title{
Acute heavy alcohol intake increases silent myocardial ischaemia in patients with stable angina pectoris
}

Juhani Rossinen, Juhani Partanen, Pekka Koskinen, Lauri Toivonen, Markku Kupari, Markku S Nieminen

\begin{abstract}
Objective-To evaluate the effect of acute alcohol ingestion on myocardial ischaemia in patients with coronary heart disease and stable angina.

Design-Randomised crossover study using fruit juice with and without ethanol. Setting-Division of cardiology in a university hospital.

Patients-20 patients with stable exertional angina and $\geqslant 50 \%$ luminal diameter narrowing of at least one major coronary artery.

Interventions-Each patient was studied on two separate days, once after administration of $1.25 \mathrm{~g}$ of ethanol per kilogram of body weight diluted to $15 \%$ in juice, and once after an equivalent volume of juice; both tests were in the evening and lasted 90 minutes. The patients were scheduled to have 8 periods of walking for $10 \mathrm{~min}$ according to a time table. An ambulatory electrocardiogram and the occurrence of anginal attacks were recorded and blood pressure and blood ethanol concentration were measured until the next morning.
\end{abstract}

Results-The blood ethanol concentration (mean (SD)) rose to $28.8 \mathrm{mmol} / \mathrm{l}(1.3$ $(0.4) \% 0)$. Alcohol raised the systolic blood pressure from 132 (16) to 141 (14) $\mathrm{mm} \mathrm{Hg}$ ( $P<0.05$ compared with juice). The mean heart rate increased from 57 (7) to 64 (8) beats/min $(P<0.05)$ for 13 hours after ethanol ingestion compared with juice. The total duration of ischaemia during the ethanol test was 3.5 (median, range 0-80) min, compared with 0 (range 0-67) min for the juice test $(P<0.05)$. The difference resulted mainly from more silent ischaemia after ethanol ingestion (2.3 $(0-80) v 0(0-67)$ min; $\mathbf{P}<0.05)$. The ST segment depression time integral increased during the ethanol test $(4 \cdot 4$ $(0-170) \mathrm{mm} \times \mathrm{min}$ ) relative to that during the juice test $(0(0-103) \mathrm{mm} \times \mathrm{min}$; $P<0.01)$ and especially during the following 13 hours after alcohol (3.5 $(0-123) \mathrm{mm} \times \mathrm{min})$ compared with juice (0 (0-67) $\mathrm{mm} \times$ min; $P<0.005)$. There were no changes in the number, duration, or ST segment depression time integral of the episodes of symptomatic angina, indicating that ethanol augmented the appearance of silent ischaemia.

Conclusions-Acute heavy ethanol drink- ing aggravates myocardial ischaemia in patients with stable angina pectoris.

(Heart 1996;75:563-567)

Keywords: silent ischaemia; ethanol; ambulatory electrocardiography.

Ethanol drinking increases the heart rate ${ }^{1}$ by stimulating the secretion of catecholamines. ${ }^{2}$ It also decreases exercise tolerance in patients with coronary heart disease, ${ }^{3}$ suggesting a worsening of the imbalance between oxygen consumption and delivery in the myocardium. Furthermore, ethanol has been shown to induce coronary artery spasms in experimental animals ${ }^{45}$ and in humans, ${ }^{67}$ but at the same time has an analgesic effect which might obscure the sensation of angina. ${ }^{89}$

Silent myocardial ischaemia is common among patients with coronary heart disease. In exercise tests over a fourth of the patients with coronary heart disease show symptomless ST segment depression in electrocardiographic recording. ${ }^{10}$ During 24-hour ambulatory electrocardiographic monitoring, about $60-70 \%$ of patients exhibit painless ischaemic episodes $^{11}$ and about $75 \%$ of all ischaemic episodes go unnoticed. ${ }^{12}$

Little is known about the effects of acute ethanol intake on the total myocardial ischaemic burden, whether symptomatic or silent. Therefore, a controlled study in patients with stable angina pectoris was conducted to assess the effects of alcohol on myocardial ischaemia.

\section{Patients and methods}

PATIENTS

Twenty patients with angiographically verified coronary heart disease and electrocardiographic evidence in a bicycle exercise test of symptomatic myocardial ischaemia were studied. Coronary angiography and left ventricular cineangiography had been carried out during the preceding four months. Patients with luminal diameter narrowing of $\geqslant 50 \%$ in at least one of the major epicardial coronary arteries and ischaemia verified by the excercise test were eligible for the study. We excluded patients with acute myocardial infarction within the preceeding month, New York Heart Association class IV symptoms, luminal diame- 
ter narrowing of $\geqslant 50 \%$ in the left main coronary artery or luminal diameter narrowing of $\geqslant 80 \%$ in all three major epicardial vessels, ejection fraction $\leqslant 25 \%$ shown by left ventricular cineangiography, previous sustained ventricular tachycardia or fibrillation, the presence of other than sinus rhythm, or a history of alcohol abuse. We also excluded patients who used digitalis and those who had ST segment depression in the resting electrocardiogram or left ventricular hypertrophy, bundle branch block, or other conditions that might confound the evaluation of ischaemia.

The study was conducted in accordance with the Helsinki Declaration for Human Research and was approved by the ethics committee of the Department of Medicine of Helsinki University Central Hospital.

\section{METHODS}

After giving their informed consent, the patients were admitted to hospital and ambulatory electrocardiographic monitoring (Holter) was started at 1200 and finished on the next day at 1210 . The patients were randomly assigned to having alcohol first or juice first in a crossover manner. The mean (SD) interval between the two tests was 7 (3) days (range 4-14). From 1700 to 1830 the patients were given $1.25 \mathrm{~g}$ of ethyl alcohol per kilogram body weight diluted to $15 \%$ in juice, yielding a total volume of $700(126) \mathrm{ml}$, or an equivalent volume of the juice alone. The dose for a person whose weight is $70 \mathrm{~kg}$ is equivalent to $2.8 \mathrm{dl}$ of $40 \%$ whisky or $9.8 \mathrm{dl}$ of $11 \%$ wine calculated from volumes and was chosen to produce distinct intoxication. This made blind administration impossible. The patients were instructed to drink at an even pace over the $1 \frac{1}{2}$ hours. Their regular daily medications were continued throughout the study. Smoking was not allowed during electrocardiographic monitoring. The episodes of anginal pain and consumption of short-acting nitrate preparations were recorded. Blood samples were taken at 1700,1900 , and 0800 for alcohol concentration measurement by gas chromatography

Table 1 Clinical characteristics of the patients

\begin{tabular}{ll}
\hline Characteristic & Value \\
\hline Age (y) & Mean 52, range 39-68 \\
Gender (F/M) & $3 / 17$ \\
Weight (kg) & Mean 84, range 54-115 \\
Duration of coronary heart disease (months) & Median 16, range 3-276 \\
Number of previous myocardial infarctions & Mean 0·7, range 0-2 \\
NYHA class: & 7 patients \\
II & 13 patients \\
III & Median 51, range 0-326 \\
Alcohol consumption (g of ethanol/week) & (2 total abstainers) \\
(51 g equals 5.7 dl of wine) &
\end{tabular}

NYHA, New York Heart Association. ${ }^{\star}$ Expressed as median, range because of skewed distribution. Self report of usual average intake.

Table 2 Characteristics of the patients' heart disease *

\begin{tabular}{ll}
\hline Characteristic & Value \\
\hline Number of patients with 1 vessel $/ 2$ vessel/3 vessel disease & $8 / 8 / 4$ \\
Average workload during the last 4 minutes of the exercise test $(W)$ & $105(39)$ \\
Heart volume in chest $x$ ray/body surface area $\left(\mathrm{ml} / \mathrm{m}^{2}\right)$ & $410(70)$ \\
Left ventricular ejection fraction $(\%)$ & $56(10)$ \\
Left ventricular end diastolic pressure $(\mathrm{mm} \mathrm{Hg})$ & $11(4)$ \\
\hline
\end{tabular}

*Values are numbers of patients or means (SD). during the alcohol experiment and at the beginning of juice ingestion.

The patients had 10 minute exercise periods at 1200,1400,1600,1800, 1900 and on the next day at 0800,1000 , and 1200 at which times they climbed 3 to 5 flight of stairs at rate that would normally provoke no angina. The length and duration of the exercises were kept constant for a patient during the whole study. The exercises during the ethanol test were accompanied by one investigator (JR).

The ambulatory electrocardiograms were recorded on tape with Marquette recorders (Marquette Electronics, Milwaukee, Wisconsin). V1 and V5-like leads were used. ${ }^{13}$ The electrocardiograms were analysed using the Marquette Series 8000 Holter analysis system without knowledge of the study phase or the patient's identity. All recordings were analysed by the same investigator (JR). ST segment depression was measured at $80 \mathrm{~ms}$ after the J point in comparison with the level of the PR interval. In cases with a persistently depressed ST segment, this level was taken as baseline. An ST segment depression $>0.1 \mathrm{mV}$ in amplitude compared with baseline and $>1$ $\mathrm{min}$ in duraton was regarded as an ischaemic episode. ST segment depression phases with an intervening period of $>1 \mathrm{~min}$ without ischaemia were calculated as separate episodes. The number of ischaemic episodes and the total duration of myocardial ischaemia and the ST depression time integral (magnitude times duration) were used to evaluate the ischaemic load. Ventricular premature complexes were detected by a semiautomatic template method (Marquette Electronics) and verified by the operator. The hourly averages of heart rate were obtained from the software report of the electrocardiographic scanner.

Blood pressure was measured using a sphygmomanometer when the Holter recording was started, at the beginning of drinking, at 1800 , before and after the walking at 1900 , and the next morning.

STATISTICAL ANALYSIS

The data are expressed as mean (SD) or median and range. Comparisons between the ethanol and juice tests were made with a Wilcoxon signed rank test or an analysis of variance with repeated measurements. ${ }^{14} \mathrm{~A} P$ value $<0.05$ with the two-tailed test was considered statistically significant. A KolmogorovSmirnov one-sample test was used to assess whether the distribution of the data was normal. If not, a logarithmic transformation was made before the analysis of variance.

\section{Results}

The clinical characteristics of the patients are given in table 1 and their heart disease variables in table 2 . Nineteen patients were taking $\beta$ blockers and long-acting nitrates, 17 aspirin, 7 calcium-channel blockers, and 4 angiotensin converting enzyme inhibitors. Nine patients had arterial hypertension; none had diabetes.

The blood ethanol concentration was 28 (8) $\mathrm{mmol} / 1$ (that is, $1.3(0.4) \% 0$ ) two hours after 
Figure 1 Heart rate responses during the ethanol and juice tests (mean heart rates are shown). The drinking period lasted from 1700 to 1830. The difference in heart rate between the ethanol and juice tests was significant from 1800 to $0700(P<0.05)$.

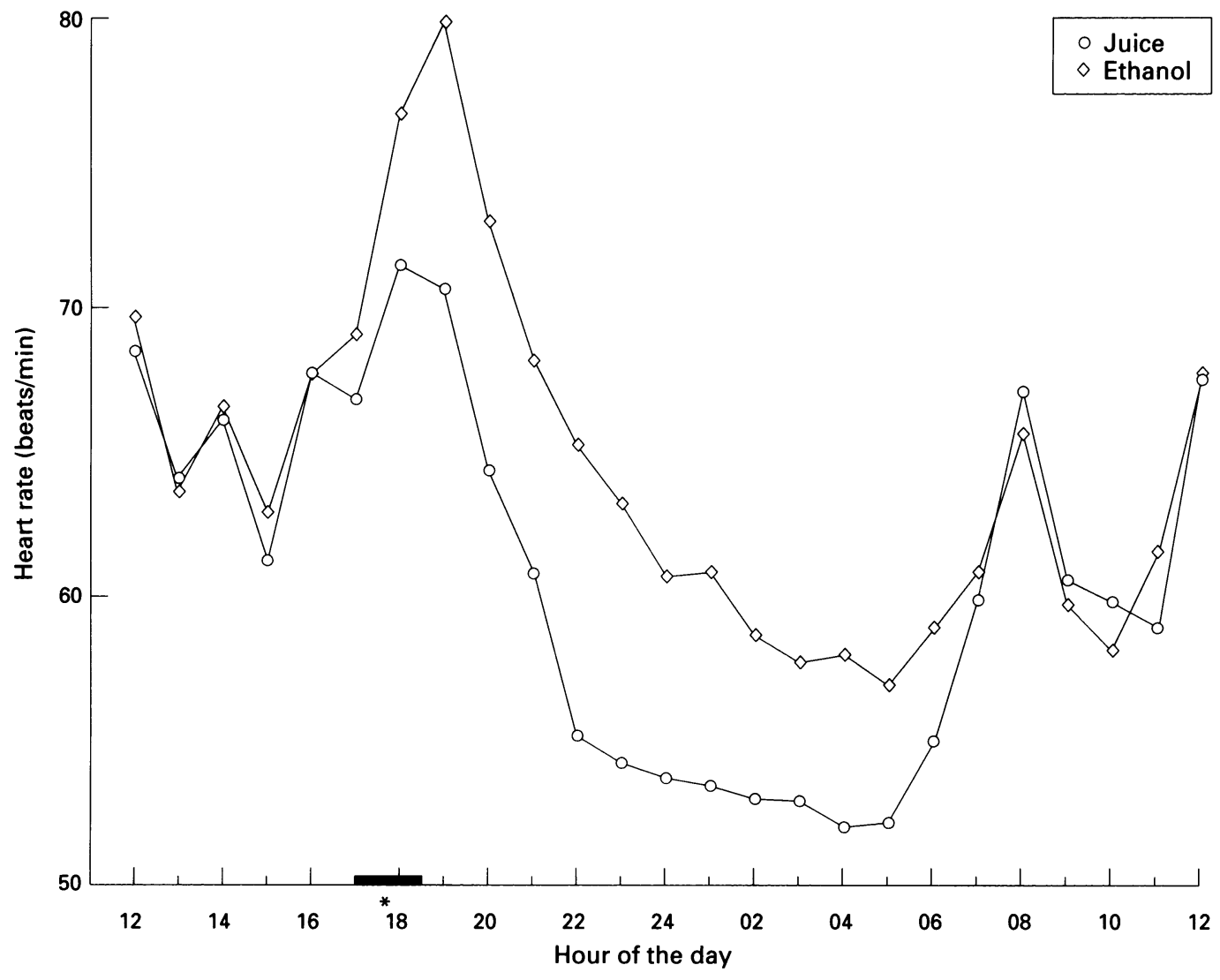

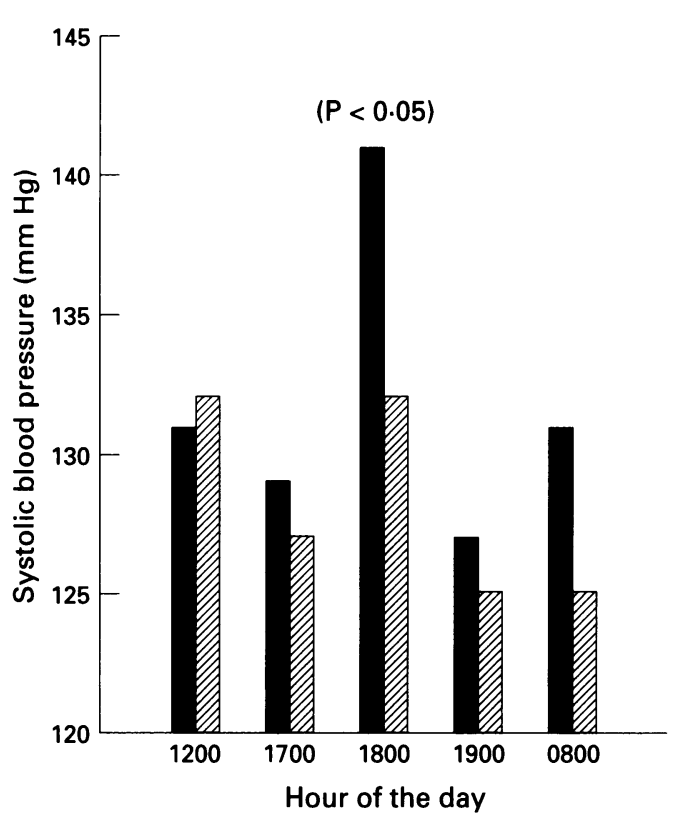

Figure 2 Systolic blood pressure during ingestion of ethanol (black columns) and juice (hatched columns). the start of drinking, and all patients showed signs of intoxication. No alcohol was detected in other blood samples.

The mean heart rate between 1800 and 0700 was significantly greater during the alcohol test than during the juice test, 64 (8) compared with 57 (7) beats/min (fig 1). Heart rate was already increased with ethanol at 1800 , an hour before taking blood for alcohol concentration measurement; and there was no indication of an effect on heart rate from the subsequent 0800 blood sampling during the alcohol test. The systolic blood pressure rose during ethanol ingestion (fig 2), but the diastolic blood pressure did not change significantly.

There were no differences between the juice and ethanol tests in blood pressure or heart rate before the drinking period.

The median number of ventricular premature complexes on the alcohol day was 4 $(0-343)$ beats and $3.5(0-632)$ beats on the juice day (NS).

Ethanol did not change the number of reported angina attacks (juice $0(0-2) v$

Table 3 Number of ischaemic attacks, episodes of angina, and consumption of short acting nitrates (median (range))

\begin{tabular}{|c|c|c|c|}
\hline & Ethanol & Fuice & $\begin{array}{l}\text { Ethanol v } \\
\text { juice }\end{array}$ \\
\hline \multirow{4}{*}{$\begin{array}{l}\text { Number of painful episodes during Holter monitoring } \\
\text { Number of ischaemic episodes during Holter monitoring } \\
\text { Consumption of short acting nitrates during whole day } \\
\text { Whole day: }\end{array}$} & $0 \quad(0-2)($ mean $0 \cdot 3)$ & $0(0-2)($ mean $0 \cdot 3)$ & NS \\
\hline & $2(0-17)$ & $0(0-24)$ & NS \\
\hline & $0 \quad(0-2)($ mean $0 \cdot 3)$ & $0(0-2)($ mean $0 \cdot 3)$ & NS \\
\hline & & & \\
\hline Total ischaemia (min) & $3.5(0-80)($ mean 12.5$)$ & $0(0-67)($ mean $6 \cdot 5)$ & $P<0.05$ \\
\hline Painful ischaemia (min) & $0 \quad(0-19)($ mean 1.8$)$ & $0(0-18)($ mean $1 \cdot 1)$ & NS \\
\hline Silent ischaemia (min) & $2 \cdot 3(0-80)$ (mean $10 \cdot 7)$ & $0(0-67)($ mean $5 \cdot 3)$ & $P<0.05$ \\
\hline 1800-0700: & & & \\
\hline Total ischaemia (min) & $3.7(0-57)($ mean 10.3$)$ & $0(0-42)($ mean $4 \cdot 5)$ & $P<0.005$ \\
\hline Painful ischaemia (min) & $0 \quad(0-19)($ mean $1 \cdot 6)$ & $0(0-18)($ mean $1 \cdot 0)$ & NS \\
\hline Silent ischaemia (min) & $2 \cdot 1(0-57)($ mean $8 \cdot 8)$ & $0(0-42)($ mean $3 \cdot 5)$ & $P<0.01$ \\
\hline
\end{tabular}


Table 4 ST Segment depression time integrals $(\mathrm{mm} \times \mathrm{min})$ (median (range))

\begin{tabular}{|c|c|c|c|}
\hline & Ethanol & Fuice & $\begin{array}{l}\text { Ethanol v } \\
\text { juice }\end{array}$ \\
\hline \multicolumn{4}{|l|}{ Whole day: } \\
\hline Total ischaemia & $4 \cdot 4(0-170)($ mean $22 \cdot 4)$ & $0(0-103)($ mean 10.6$)$ & $P<0.01$ \\
\hline Painful ischaemia & $0 \quad(0-44)($ mean 3.0$)$ & $0(0-37)($ mean $2 \cdot 1)$ & NS \\
\hline Silent ischaemia & $2 \cdot 7(0-170)($ mean $19 \cdot 5)$ & $0(0-103)($ mean $8 \cdot 5)$ & $P<0.05$ \\
\hline \multicolumn{4}{|l|}{ 1800-0700: } \\
\hline Total ischaemia & $3 \cdot 5(0-123)($ mean $18 \cdot 1)$ & $0(0-67)($ mean $7 \cdot 4)$ & $P<0.005$ \\
\hline Painful ischaemia & $0 \quad(0-44)$ (mean $2 \cdot 7)$ & $0(0-40)($ mean $2 \cdot 1)$ & NS \\
\hline Silent ischaemia & $2 \cdot 7(0-123)($ mean $15 \cdot 4)$ & $0(0-67)($ mean $5 \cdot 3)$ & $P<0.01$ \\
\hline
\end{tabular}

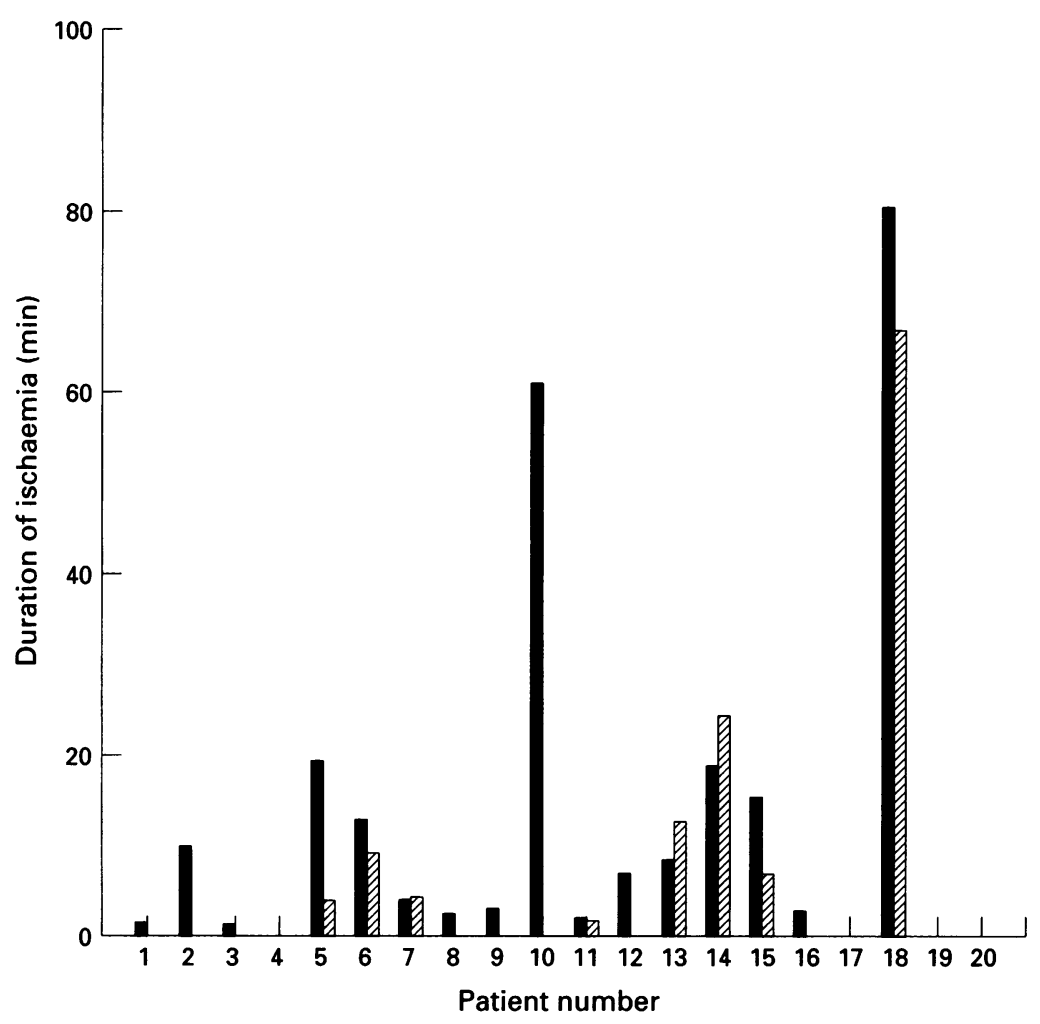

Figure 3 Duration of ischaemia in individual patients during the ethanol (black columns) and juice (hatched columns) tests. The ingestion of ethanol increased ischaemia (median 3.5, range 0-80) compared with the ingestion of juice (median 0, range 0-67; $P<0.05)$. aggravation of ischaemia. Decreased pain perception, in accord with the analgesic effect of ethanol, may have helped to prevent patient awareness of the episodes.

Previous studies support our finding of increased silent ischaemia. Orlando et $a l^{15}$ evaluated the effect of ethanol on exercise performance until the appearance of angina pectoris in patients with coronary artery disease in a study in which the achieved ethanol concentration was lower $(1.0(0.15) \%)$ than in our study. Compared with control periods, the mean exercise time until angina was decreased after ethanol. Interestingly, there was more ischaemic ST segment depression after ethanol than after juice in the exercise level when angina was experienced, and the magnitude of ST segment depression increased with ethanol dose. This supports the hypothesis of increased silent myocardial ischaemia induced by ethanol. On the other hand, Pirwitz et al ${ }^{16}$ have recently reported that intravenous ethanol induces epicardial coronary arterial vasodilatation. In their study, however, the rate-pressure product did not increase, perhaps because the blood ethanol concentration achieved $(0.86(0.23) \% 0)$ was lower than in our study. The increase in heart rate and blood pressure, and thus oxygen consumption, probably outweighs the possible vasodilator action of ethanol in the higher, rather toxic concentrations, used in our present study.

Ethanol depresses myocardial contraction in patients with heart disease. ${ }^{17} 18$ However, Greenberg et al have observed a decrease in vascular resistance after ethanol that counterbalances the deterioration in cardiac performance. ${ }^{19}$ Our study gives no insight into whether left ventricular volume or myocardial contractility changed during ethanol ingestion. Thus it is also possible that by increasing left ventricular volume, ethanol might increase myocardial oxygen consumption.

In patients with ischaemic heart disease verified by coronary angiography, ST segment depression detected by ambulatory ECG monitoring is likely to indicate ischaemia. ${ }^{12}$ This suggests that the changes observed in our patients reflected a real myocardial oxygen deficit. The ST depression time integral is a valid indicator of ischaemia because the magnitude of ST segment depression in the Holter recording has been shown to correlate well with the severity of ischaemia demonstarted by an exercise test. ${ }^{20}$ Admittedly, the ST segment is affected not only by myocardial ischaemia, but also by ventricular hypertrophy, conduction disturbances, and pharmacological agents. ${ }^{13}$ Patients with these conditions were, however, excluded from the study.

All patients except one were taking $\beta$ blockers. The mean rise of heart rate due to alcohol between 1800 and 0700 was only 7 (2) beats/min despite the high ethanol concentration $(28(8) \mathrm{mmol} / \mathrm{l}(1.3(0.36) \% 0))$. The pulse frequency would probably have increased more after ethanol ingestion without $\beta$ blockade, ${ }^{21}$ and thus ischaemia could have been even more marked without this treatment.

Moderate consumption of alcohol has been
The study shows that acute ethanol ingestion provokes asymptomatic myocardial ischaemia. The observed increases in both systolic blood pressure and heart rate which reflect myocardial oxygen consumption seem to explain the 
shown to reduce mortality in epidemiological studies. ${ }^{22}$ This contrasts with the harmful effects on ischaemia observed here. The daily ethanol dose associated with reduced mortality has, however, been much smaller than that used in the present experiment, which lead to signs of acute intoxication. Furthermore, there is only limited information on the influence of ethanol on mortality in patients with coronary heart disease.

The study has potential limitations: day-today variation in myocardial ischaemia is considerable in patients with coronary heart disease. ${ }^{23}$ Our finding of ethanol-induced ischaemia, however, seems reliable because most of the increase in ischaemia occurred during ethanol intoxication and shortly after it. Although complete blinding is impossible in ethanol studies, the analysis of Holter recordings was blinded. The hospital ward does not represent the usual drinking circumstances of the patients and the mode of exercise performed can be questioned because quantification of the exercise level is difficult in floor climbing. It was, however, preferred over the conventional bicycle ergometry because it corresponds more closely to the exercise likely in usual drinking conditions. The amount of ethanol the patients consumed in this study was rather large and its relevance to lower doses is unknown: it should not be concluded from these results that social intake of 1 or 2 drinks causes ischaemia in patients with coronary heart disease. On the other hand, large amounts of alcohol are commonly drunk in Finland. ${ }^{24}$ Our results show that consuming alcohol in such amounts can provoke silent myocardial ischaemia in patients with coronary heart disease and should be discouraged.

1 Kupari M. Acute cardiovascular effects of ethanol, a controlled non-invasive study. Br Heart $f$ 1983;49:174-82.

2 Perman ES. The effect of ethyl alcohol on the secretion from the adrenal medulla in man. Acta Physiol Scand 1958;44:241-7.

3 Regan TJ. Regional circulatory responses to alcohol and its congeners. Fed Proc 1982;41:2438-42.
4 Rogers PJ, Bove AA. Epicardial coronary artery constriction with intravenous ethanol. Int $f$ Cardiol 1989;22: 301-10.

5 Altura BM, Altura BT, Carella A. Ethanol produces coronary vasospasm: evidence for a direct action of ethanol nary vasospasm: evidence for a direct action of
on vascular muscle. $\mathrm{Br} \mathcal{f}$ Pharmac 1983;78:260-2.

6 Kashima T, Tanaka H, Arikawa K, Ariyama T. Variant angina induced by alcohol ingestion. Angiology 1982;33: angina

7 Altura BM, Altura BT. Microvascular and vascular smooth muscle actions of ethanol, acetaldehyde, and acetate. Fed muscle actions of ethan
Proc 1982;41:2447-51.

8 Ritchie JM. The aliphatic alcohols. In: Gilman AG, Goodman LS, Rall TW, Murad F, eds. Pharmacological basis of therapeutics. New York: MacMillan.1985;372-86.

9 James MF, Duthie AM, Duffy BL, McKeag AM, Rice CP. Analgesic effect of ethyl alcohol. Br f Anaesth 1978; 50:139-41.

10 Weiner DA, Ryan TJ, McCabe CH, et al. Significance of silent myocardial ischemia during exercise testing in patient with coronary artery disease. Am $\mathcal{F}$ Cardiol 1987; 59:725-9.

11 Pepine CJ. Is silent ischemia a treatable risk factor in patients with angina pectoris? Circulation 1990;82 (suppl II):II-135-II-142.

12 Deanfield JE, Selwyn AP, Chierchia S, et al. Myocardial ischemia during daily life in patients with stable angina pectoris: its relation to symptoms and heart rate changes. Lancet 1983;2:753-8.

13 Kennedy HL. Ambulatory electrocardiography strategies used in assessing silent myocardial ischaemia. Eur Heart $\mathcal{f}$ 1988;9 (suppl N):70-7.

14 Hills $M$, Armitage P. The two-period cross-over clinical trial. Br f Clin Pharmac 1979;8:7-20.

15 Orlando J, Aronow WS, Cassidy J, Prakash R. Effect of ethanol on angina pectoris. Ann Intern Med 1976;84: 652-5.

16 Pirwitz MJ, Lange RA, Willard JE, et al. Effects of intravenous ethanol on diameter of epicardial coronary arteries. venous ethanol on diameter 7 Cardiol 1995;75:77-9.

17 Gould L, Zahir M, DeMartino A, Comprecht RF. Cardiac effects of a cocktail. $¥ A M A$ 1971;218:1799-802.

18 Conway $\mathrm{N}$. Haemodynamic effects of ethyl alcohol in Conway N. Haemodynamic effects of ethyl alcohol in
patients with coronary heart disease. $B r$ Heart $\mathcal{f} 1968 ; 30$ : 638-44.

19 Greenberg BH, Schutz R, Grunkemeier GL, Griswold H. Acute effects of alcohol in patients with congestive heart failure. Ann Intern Med 1982;97:171-5.

20 Detry JM, Fesler R, Berckmans T, Leclercq P. A new semiautomated algorithm to quantify holter-detected myocardial ischemia: preliminary experience in the Trimetazidine European Multicenter Trial (TEMS), Cardiovasc Drugs Ther 1990;4:841-6.

21 Kupari M, Heikkilä J, Tolppanen E-M, Nieminen MS Ylikahri R. Acute effects of alcohol, beta blockade, and their combinations on left ventricular function and hemodynamics in normal man. Eur Heart $¥ 1983 ; 4$ : 463-71.

22 Doll R, Peto R, Hall E, Wheatley K, Gray R. Mortality in relation to consumption of alcohol: 13 years' observations on male British doctors. Br Med f 1994;309:911-8.

23 Tzivoni D, Gavish A, Benhorin J, et al. Day-to-day variability of myocardial ischemic episodes in coronary artery disease. Am 7 Cardiol 1987;60:1003-5.

24 Suhonen O, Aromaa A, Reunanen A, Knekt P. Alcohol consumption and sudden coronary death in middle-aged Finnish men. Acta Med Scand 1987;221:335-41. 